\title{
Correspondence
}

\section{CATASTROPHIC MIGRAINE}

\section{To the Editor}

As a follow up to the paper by Fisher, ' we wish to report two further cases quite similar to what he refers to as "catastrophic migraine"'.

Case 1 A 28 year old caucasian woman, with a history of common migraine for many years and use of oral contraception for 5 months, experienced a sharp rapidly worsening headache spreading from the left fronto-temporal area to the whole scalp. During the night, she developed right hemi-anopia, paresthesiae and had difficulty finding words. The following day the headache was still sharp and associated with photo-phobia. There was a right facial weakness and a homonymous hemi-anopia $(\mathrm{HH})$, but no meningeal signs, hypaesthesia or aphasia. A CT scan with contrast enhancement (Figure 1) performed on the fifth day showed a low density area in the deep and superficial territory of the left posterior cerebral artery (PCA). Regional cerebral blood flow ( $\mathrm{Xe}^{123}$ inhalation) was impaired in the left temporo-occipital region. Clinical and ultrasonic evaluation of cervical vessels, electrocardiogram (EKG) and 24h EKG monitoring were normal. Angiography was not performed. A part from migraine and oral contraceptive medication, there were no risk factors. Extensive evaluation of hemostasis was normal. The frontal headache decreased after 3 days, but the neurological deficits remained unchanged.

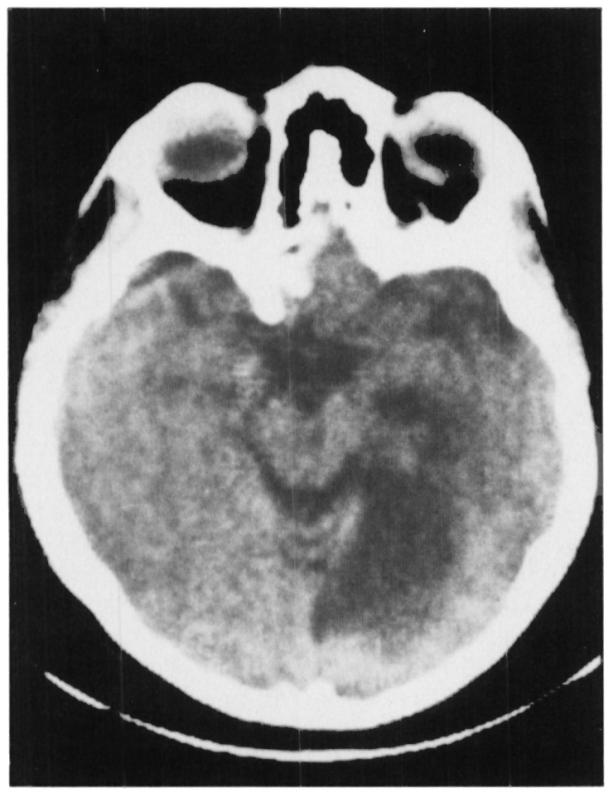

Figure I-Case I.

Case 2 A 48 year old caucasian woman had a history of accompanied migraine (facio-brachial paresthesiae and scintillating scotoma) and end-stage polycystic renal disease (creatinine: $707 \mu \mathrm{Mol} / \mathrm{l}$ ). In January 1984 , she suddenly developed a left $\mathrm{HH}$ which partially improved later. A CT scan showed a low density area in the right PCA territory. In February 1985, she developed a right scintillating scotoma and then right $\mathrm{HH}$ and paresthesiae of the index finger. One hour later, a left fronto-orbital throbbing pain spread to the whole scalp, without disappearance of the right $\mathrm{HH}$. The next day she was admitted to hospital after a generalized convulsive seizure. She still complained of a severe, diffuse and throbbing headache. Examination disclosed a left $\mathrm{HH}$, a right superior quandrantanopia and a central scotoma. Left tendon reflexes were brisk. A CT scan without contrast (Figure 2) showed a low density area in right occipital and left parieto-occipital regions. Blood pressure was 190/100, cardiac examination, EKG, creatine kinase levels and clinical examination of cervical arteries were all normal. Angiography was not performed. The headache disappeared within 4 days. Clinical signs remained otherwise unchanged.

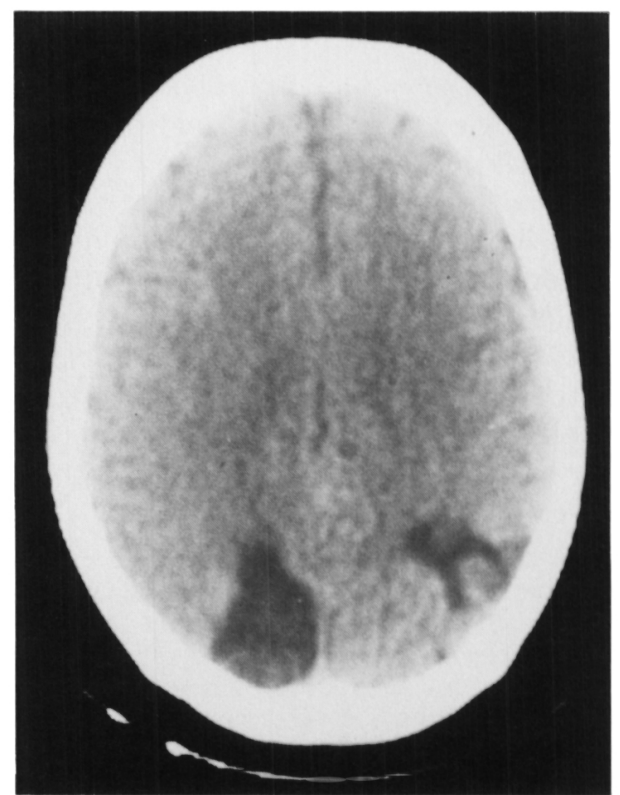

Figure 2 - Case 2

Our two patients are very similar to Fisher's cases ${ }^{1}$ with sudden onset in migrainous women of one or more ischemic strokes in the PCA territory. In our first case as in two of Fisher's cases, superficial and deep PCA territories were involved. Migrainous background and migrainous headache at onset prompted Fisher to coin the term "catastrophic migraine" or "cataclysmic migraine". However, migrainous diathesis is often not the only causative factor. Four of Fisher's 10 cases were receiving or had received hormonal therapy (oral contraception in 3 cases, estrogens alone in I case). One woman was pregnant, another was a heavy smoker and one had mitral valve prolapse. Our first case was receiving oral contraception, the second had end-stage polycycstic renal disease with hypertension. Furthermore, this "catastrophic migraine" seems very rare in comparison to the usual forms of migrainous disease. We consider that vascular disease is necessary prior to the onset of a stroke, which may involve the middle cerebral artery territory as well as PCA supply. ${ }^{2-3}$ In the first instance, it is possible that a stroke, whatever its etiology may be, causes a migrainous attack in a susceptible individual ${ }^{2}$. On the other hand, the peculiar occurrence of stroke in the PCA territory may be due to hemodynamic disturbances induced in that region by the migrainous attack. ${ }^{4}$

Pascal Benoit Alain Destee Pierre Warot Lille, France

\section{REFERENCES}

1. Fisher CM. Unusual vascular events in the territory of the posterior cerebral artery. Can J Neurol Sci 1986; 13: 1-7.

2. Bartleson JD. Transient and persistent neurological manifestations of migraine. Stroke 1984; 15: 383-386.

3. Spaccavento LJ, Solomon GD. Migraine as an etiology of stroke in young adults. Headache $1984 ; 24: 19-22$.

4. Skinhøj E. Hemodynamic studies within the brain during migraine. Arch Neurol 1973; 29: 95-98. 